\title{
Intestinal torsion causing chylous ascites: a rare occurrence
}

\author{
Ye Xin Koh ${ }^{1}$, Mrcs, Jack Kian Chng ${ }^{1}$, Mrcs, Hwei Ching Tan ${ }^{1}$, Mrcs, Alexander Yaw Fui Chung ${ }^{1}$, FRcs
}

ABSTRACT Intestinal torsion and chylous ascites are very rarely associated. We present the case of a 19-year-old man who presented with acute abdomen. Computed tomography of his abdomen showed features suggestive of intestinal torsion. Chylous ascites was incidentally discovered on exploratory laparotomy. The chylous fluid was drained, the small bowel detorted and the coloduodenal adhesion band taken down. The patient's retroperitoneum was explored to exclude occult masses and malformations of the lymphatics. Post surgery, the patient recovered uneventfully. In this case, we postulate that intestinal malrotation had caused the obstruction of the lymphatic flow from the mesenteric lymphatic channels, leading to the exudation of chyle, which then resulted in the accumulation of chylous fluid in the peritoneal cavity. It is important to exclude the more common causes of atraumatic chylous ascites, such as enlarged retroperitoneal lymph nodes or lymphatic malformations.

Keywords: chylous ascites, intestinal malrotation

\section{INTRODUCTION}

Since the first case of acute chylous peritonitis described by Renner $^{(1)}$ in 1910, there have been few case reports about chylous peritonitis. Likewise, intestinal malrotation presenting in adults is a rare occurrence that has been limited to only a few case reports and small case series. ${ }^{(2,3)}$ However, these two conditions are very rarely associated. Here, we present a unique case of intestinal malrotation with development of chylous ascites, presenting as acute abdomen. If chylous ascites is found intraoperatively, specific measures should be taken to exclude pathologies that are commonly known to cause chylous ascites.

\section{CASE REPORT}

A 19-year-old Chinese man presented with severe acute periumbilical pain associated with vomiting for one day. The patient did not have any history of trauma or abdominal surgery. On physical examination, he was afebrile and haemodynamically stable. His abdomen was guarded, with marked tenderness in the periumbilical region. His abdomen appeared distended and there was a dullness on percussion of the flanks. Laboratory investigations were as follows: haemoglobin $16.1 \mathrm{~g} / \mathrm{dL}$; total leucocyte count $12.88 \times 10^{9} / \mathrm{L}$; amylase $39 \mathrm{U} / \mathrm{L}$; lipase $21 \mathrm{U} / \mathrm{L}$. Computed tomography (CT) showed a whirl-like appearance, with a collapsed bowel around the superior mesenteric artery (Fig. 1). There was also an abrupt cut-off of the superior mesenteric vein just distal to the splenoportal confluence (Fig. 2). This was suggestive of intestinal torsion.

On exploratory laparotomy, two litres of chylous fluid was drained from the peritoneal cavity. The small bowel mesentery was torted around an adhesion band between the transverse colon and duodenum. The small bowel mesentery was found to be long and narrow, and the position of the caecum was to the right and more cranial than usual. The bowel was well-perfused,

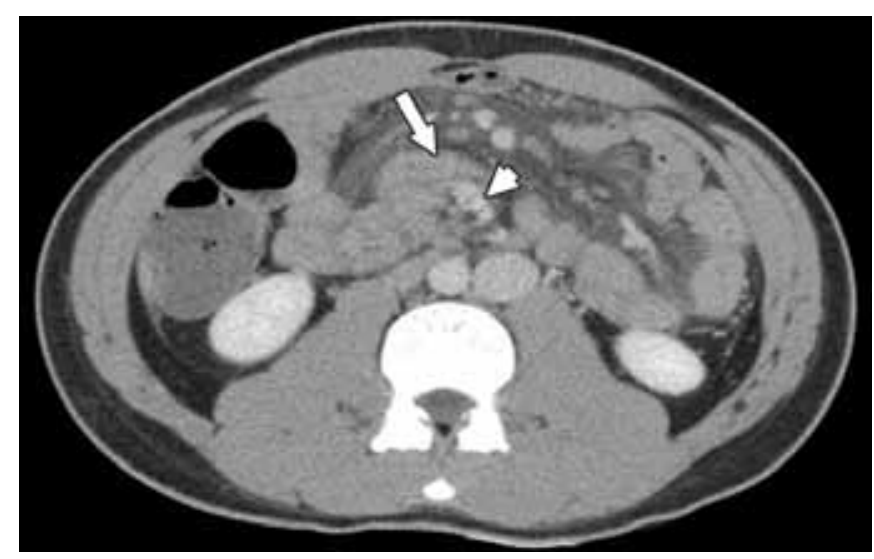

Fig. 1 CT image shows a whirl sign with a collapsed bowel (arrow) around the superior mesenteric vessels (arrowhead).

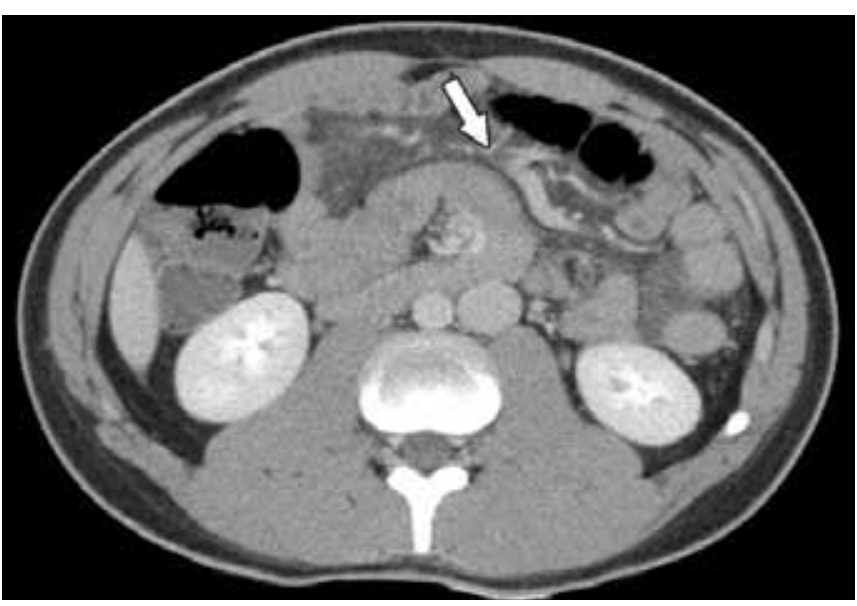

Fig. 2 CT image shows an abrupt venous cut-off (arrow) at the superior mesenteric vein just distal to the splenoportal confluence.

pink and only mildly dilated. Superior mesenteric arterial pulsations were prominent.

The bowel was detorted in a counterclockwise manner and the coloduodenal adhesion band was taken down. Adhesions

${ }^{1}$ Department of General Surgery, Singapore General Hospital, Singapore

Correspondence: Dr Koh Ye Xin, Resident, Department of General Surgery, Singapore General Hospital, Outram Road, Singapore 169608. yexin_koh@hotmail.com 
around the superior mesenteric artery were also taken down so as to broaden the base of the mesentery. The palpable mesenteric lymph nodes were biopsied. Exploration of the retroperitoneum showed no obvious malformations of the lymphatics. Extensive lavage of the abdominal cavity was performed until the return fluid was clear. At the end of the operation, the bowel remained healthy and well-perfused (Fig. 3). The patient's peritoneal fluid triglyceride was $504 \mathrm{mg} / \mathrm{dL}$ and serum microglobulin was $1,232 \mathrm{UG} / \mathrm{L}$. Culture and cytology of the peritoneal fluid was negative for tuberculosis and malignancy, respectively. Histology of the biopsied lymph node revealed benign reactive hyperplasia.

Postoperatively, the patient was started on a low-fat, highprotein diet. Examination on postoperative Day 4 showed no chylous fluid in the abdominal drain, and the drain was removed on postoperative Day 5. He was discharged well and followed up as an outpatient three months after hospitalisation. He had no complaints of abdominal pain and no problems with resuming a normal diet. No further imaging investigations were performed as the patient was asymptomatic.

\section{DISCUSSION}

Although intestinal malrotation is a well-recognised disease in the paediatric group, it is not well described in adults. Rotational abnormalities of the intestine result from the failure of normal embryologic rotation and fixation of the bowel mesentery. Various anatomical abnormalities have been described, including nonrotation, reversed rotation and varying severities of irregular rotation. ${ }^{(4)}$ In our case, even though the operative findings were not classical of malrotation, it bore the characteristics of irregular rotation, which included a long and narrow mesentery, the presence of adhesion bands, an abnormally high position of the caecum (indicating failure of descent), and clockwise rotation of the bowel. However, CT revealed classic signs of intestinal malrotation - a whirl-like appearance of the collapsed small bowel encircling the superior mesenteric artery. The use of a 'whirl sign' in the diagnosis of small bowel volvulus had been described by De Korte et al. ${ }^{(5)}$ There was also an abrupt cut-off of the superior mesenteric vein at the area of the torsion, typical of a 'venous cut-off sign', which has been reported by $\mathrm{Ho}^{(6)}$ to be a more apparent identifying feature of intestinal torsion.

The differential diagnoses for chyle are pseudochyle and pus, and it is of significant importance to distinguish these entities. Chyle is distinguished by its odourless milky appearance, which is similar to peripheral lymphatic fluid. Its white cell count averages $5,000 / \mathrm{mL}$ and it is sterile in culture. The triglyceride concentration of chyle is typically 2-8 times that of plasma (> $200 \mathrm{mg} / \mathrm{dL}$ ) and the fat globules present in chyle stain positive with Sudan red stain. ${ }^{(7)}$ Pseudochyle and pus are opalescent chylous fluids, which are found in patients with cellular degeneration due to bacterial peritonitis or carcinomatosis peritonei. In such cases, the triglyceride concentration is usually not elevated. Furthermore, pseudochyle and pus do not have similar biochemical properties as chyle. In our case, the milky

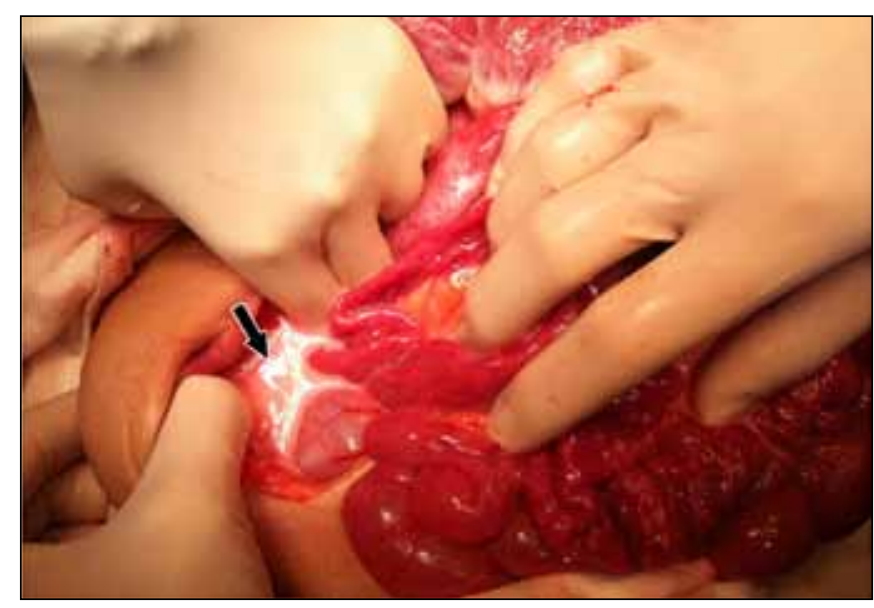

Fig. 3 Photograph taken at laparotomy after detorsion of small bowel malrotation shows chylous fluid (arrow), and a healthy, well-perfused bowel.

fluid from the patient showed marked triglyceride elevation, which was diagnostic of chylous ascites. In normal physiology, the gut lymphatics carry converted long-chain triglycerides from the intestine to the vascular system via the thoracic collecting duct at rates of $50-200 \mathrm{~mL} / \mathrm{kg} / \mathrm{hr}{ }^{(8)}$ Hence, in cases of acute lymphatic obstruction, rapid accumulation of chyle can occur.

A review article by Steinemann et al on the causes of atraumatic chylous ascites showed that $84 \%$ of paediatric cases were a result of congential lymphatic anomalies. ${ }^{(9)}$ In adults, however, atraumatic chylous ascites is more commonly due to malignant tumours, namely lymphomas. Other important causes in adults include liver cirrhosis, mycobacterial infections and abdominal surgery, such as those associated with aortic aneurysm repair and surgery on retroperitoneal structures such as lymph nodes. ${ }^{(10)}$ In our case, we postulate that the intestinal malrotation caused the obstruction of the lymphatic flow, leading to the exudation of chyle and the eventual accumulation of chylous fluid. Disruptions of the lymphatic flow causing chylous ascites was first described by Kinmonth ${ }^{(11)}$ in 1976.

The optimal management of chylous ascites depends on its underlying aetiology. Lymphagiography and lymphoscintigraphy would aid in the evaluation of underlying lymphatic malformations. $^{(12)}$ If lymphatic malformations are found, surgical management is straightforward and correction can be made by ligation of leaking lymphatics or resection of lesions obstructing the lymphatic flow. However, in our case, this was not possible due to the acute presentation of abdominal pain. Furthermore, lymphagiography and lymphoscintigraphy are usually reserved for non-emergency situations. Intraoperative exploration of the retroperitoneum was then mandatory so as to exclude occult lesions. In our case, the classical management principles for surgical treatment of intestinal malrotation were applied. Counterclockwise detorsion of the bowel, division of the coloduodenal band and division of adhesions around the superior mesenteric artery to broaden the base of the mesentery were performed. ${ }^{(13)}$ Importantly, there were no lymphatic malformations, enlarged lymph nodes or retroperitoneal tumours at the root 
of the mesentery and retroperitoneum, which might have obstructed the lymphatic flow. Hence, the cause of the chylous ascites could be confidently attributed to intestinal malrotation, which resulted in temporary lymphatic obstruction. Other supportive measures to decrease the rate of chyle formation include total parenteral nutrition to achieve complete bowel rest, and a low-fat, high-protein diet to decrease lymph flow in the major lymphatic tracts. ${ }^{(14)}$ As our patient's underlying cause of mesenteric torsion was treated, he did not require complete bowel rest with total parenteral nutrition.

As illustrated in our case, it is important to recognise that intestinal malrotation can result in chylous ascites. However, appropriate measures should be taken to exclude the common underlying conditions associated with the development of chylous ascites. In the majority of patients who presented with intestinal malrotation and chylous ascites, surgical correction of intestinal malrotation and adequate peritoneal lavage resulted in favourable outcomes with no recurrence of the problems. ${ }^{(15,16)}$ This was also demonstrated in our case, where the correction of the underlying intestinal malrotation was essential for the treatment of malrotation-induced chylous ascites.

\section{REFERENCES}

1. Renner A. [Chylus als Bruchwasser beim eingeklemmten Bruch]. Beitr Klin Chir 1910; 70:695-8. German.

2. Hsu SD, Yu JC, Chou SJ, et al. Midgut volvulus in an adult with congenital malrotation. Am J Surg 2008; 195:705-7.
3. Fu T, Tong WD, He YJ, et al. Surgical management of intestinal malrotation in adults. World J Surg 2007; 31:1797-803.

4. Lampl B, Levin TL, Berdon WE, Cowles RA. Malrotation and midgut volvulus: a historical review and current controversies in diagnosis and management. Pediatr Radiol 2009; 39:359-66.

5. De Korte N, Grutters CT, Snellen JP. Small bowel volvulus diagnosed by CT "whirl sign". J Gastrointest Surg 2008 12:1469-70.

6. Ho YC. "Venous cut-off sign" as an adjunct to the "whirl sign" in recognizing acute small bowel volvulus via CT scan. J Gastrointest Surg 2012; 16:2005-6.

7. Ward PC. Interpretation of ascitic fluid data. Postgrad Med 1982; 71:171-3.

8. Madding GF, McLaughlin RF, McLaughlin RF Jr. Acute chylous peritonitis. Ann Surg 1958; 147:419-22.

9. Steinemann DC, Dindo D, Clavien PA, Nocito A. Atraumatic chylous ascites: systematic review on symptoms and causes. J Am Coll Surg 2011; 212:899-905.

10. Ohri SK, Patel T, Desa LA, Spencer J. The management of postoperative chylous ascites. A case report and literature review. J Clin Gastroenterol 1990; 12:693-7.

11. Kinmonth JB. Disorders of the circulation of chyle. J Cardiovasc Surg (Torino) 1976; 17:329-39.

12. Losanoff JE, Richman BW, Jones JW. Chylous ascites. Am J Gastroenterol 2003; 98:219-20.

13. Fu T, Tong WD, He YJ, et al. Surgical management of intestinal malrotation in adults. World J Surg 2007; 31:1797-803.

14. Aalami OO, Allen DB, Organ $\mathrm{CH}$ Jr. Chylous ascites: a collective review. Surgery 2000; 128:761-78.

15. Vettoretto $N$, Odeh $M$, Romessis $M$, et al. Acute abdomen from chylous peritonitis: a surgical diagnosis. Case report and literature review. Eur Surg Res 2008; 41:54-7.

16. Ozgüç H, Narmanlı M, Keskin MK. Acute chylous peritonitis: Report of a case. Int J Surg Case Rep 2013;4:419-21. 